\title{
A Case-Control Study on the Risk Factors for Hepatocellular Carcinoma in Fukuoka, Japan
}

\author{
Keitaro Tanaka and Tomio Hirohata \\ Department of Public Health, Kyushu University School of Medicine, Fukuoka, Japan
}

\begin{abstract}
We carried out a case-control study of hepatocellular carcinoma (HCC) including 204 patients with $\mathrm{HCC}$ and 410 control subjects in Fukuoka prefecture, where HCC risk is among the highest in Japan. The possible risk factors examined were: a) chronic hepatitis B virus (HBV) infection, b) alcohol consumption and cigarette smoking, c) a past history of blood transfusion and hepatitis $\mathrm{C}$ virus (HCV) infection. Chronic HBV infection was strongly associated with the development of HCC (relative risk, RR =14.6). Heavy drinkers experienced about a two-fold risk increase, yet a relationship between cigarette smoking and HCC was not evident. Blood recipients showed a significantly increased RR of 3.0. Serum antibodies to HCV (antiHCV) were detected by both enzyme-linked immunosorbent assay and recombinant immunoblot assay in $51 \%$ of HCCs and $3 \%$ of the controls; the RR (and 95\% confidence interval) for positive anti-HCV was calculated as 52.3 (23.9-114.3). Among male patients with HCC, the anti-HCV rates were very high in blood recipients $(68 \%)$, heavy drinkers $(62 \%)$ and those who had no identifiable risk factors $(75 \%)$, indicating the possible transmission of $\mathrm{HCV}$ via routes other than blood transfusion. We estimated the population attributable risk of $\mathrm{HCC}$ at $17 \%$ for chronic $\mathrm{HBV}$ infection, $13 \%$ for heavy drinking, and $49 \%$ for HCV infection in Fukuoka. Further investigations are still needed to identify the other infectious routes of HCV besides transfusion.
\end{abstract}

Hepatocellular carcinoma, Hepatitis B virus, Hepatitis C virus, Alcohol, Smoking, Case-control study

\section{INTRODUCTION}

In Japan, figures for mortality from primary liver cancer (PLC) show a characteristic pattern from the view of descriptive epidemiology. First, among the male population, the ageadjusted mortality rate has been sharply increasing over the past decade, whereas no such trend has been observed among the female population. ${ }^{(1)}$ This phenomenon probably reflects the real 
increase of the occurrence of PLC in Japanese men and the possible importance of factors more common in men than in women. Second, the geographic distribution of PLC mortality illustrates the presence of substantially higher rates in western Japan, especially in northern Kyushu Island, where our study area, Fukuoka prefecture, is situated. Since 1989, in this prefecture, PLC has been ranked as the first and third leading cause of site-specific cancer deaths in men and women, respectively.

More than $90 \%$ of PLCs in Japan are known to be hepatocellular carcinomas (HCCs). ${ }^{(2)}$ The potential determinants of HCC among the Japanese population include the following factors. A) Chronic hepatitis B virus (HBV) infection: It has been claimed that chronic HBV infection accounts for around $40 \%$ of all HCC occurrence in Japan. However, the Liver Cancer Study Group of Japan has reported a considerable decline in the prevalence of HBV carriers among patients with HCC over the last two decades (Table 1). ${ }^{(2,3)}$ Factors other than chronic HBV infection may therefore be playing an increasingly important role. B) Life styles, particularly alcohol consumption and cigarette smoking: Alcohol is a well-known hepatotoxic agent, and heavy drinking has long been implicated in the etiology of HCC. ${ }^{(4)}$ Also, recent epidemiologic studies have suggested a possible association of cigarette smoking with HCC. (5- $^{\text {- }}$ ${ }^{8)}$ These habits are common among Japanese men and so deserve attention in the light of the observed increase of male PLCs. C) Past history of blood transfusion and hepatitis $\mathrm{C}$ virus (HCV) infection: Several clinical investigations have also revealed the development of HCC from patients with post-transfusion non-A, non-B hepatitis, ${ }^{(9)}$ and thus a past history of blood transfusion has been suspected as a potential risk factor for HCC. In 1989, Choo et al. reported the discovery of complementary DNA, presumably derived from a blood-borne non-A, non-B hepatitis virus. ${ }^{(10)}$ They designated the virus as HCV and devised an assay for serum antibodies to HCV (anti-HCV). ${ }^{(11)}$ Data on the results from the assay demonstrated the high prevalence of anti-HCV among HCC patients, ${ }^{(12.13)}$ and the possible association between $\mathrm{HCC}$ and HCV infection has been highlighted.

We conducted a case-control study of HCC in Fukuoka prefecture in relation to the above factors, based on a detailed interview survey as well as a serological determination of HBV and HCV infection.

Table 1 Trends in prevalence of serum HBsAg among HCC patients reported by the Liver Cancer Study Group of Japan

\begin{tabular}{cc}
\hline Period (Years) & Prevalence of HBsAg \\
\hline $1968-1977$ & $40.7 \%$ \\
$1978-1979$ & $34.0 \%$ \\
$1980-1981$ & $31.4 \%$ \\
$1982-1983$ & $27.5 \%$ \\
$1984-1985$ & $24.6 \%$ \\
\hline
\end{tabular}




\section{SUBJECTS AND METHODS}

The details of the present study have been described elsewhere. ${ }^{(14-16)}$ In brief, the cases comprised of 204 Japanese patients with HCC who were admitted to Kyushu University Hospital from December 1985 to June 1989. The selection criteria for cases were: a) those diagnosed as HCC within 1 year prior to identification; b) those aged 40 to 69 years; c) residents of either Fukuoka prefecture or Saga prefecture (neighboring Fukuoka prefecture) and of Japanese nationality. Of the 204 cases, 195 were residents of Fukuoka prefecture, while 9 were from Saga prefecture.

The controls consisted of 410 health examinees aged 40 to 69 years who visited the Hakata Public Health Center located near Kyushu University Hospital between January 1985 and July 1989 . We selected controls so that their distribution by sex and age was as close as possible to that of the cases. Those who suffered from definite chronic liver diseases such as chronic hepatitis and liver cirrhosis were excluded. The demographic characteristics of the study subjects are shown in Table 2.

Table 2 Distribution of study subjects by demographic characteristics

\begin{tabular}{|c|c|c|c|c|}
\hline \multirow{2}{*}{ Characteristic } & \multicolumn{2}{|c|}{ Males } & \multicolumn{2}{|c|}{ Females } \\
\hline & HCC & Controls & HCC & Controls \\
\hline No. of subjects & 168 & 291 & 36 & 119 \\
\hline \multicolumn{5}{|l|}{ Age class } \\
\hline $40-49$ & 14 & 52 & 5 & 15 \\
\hline $50-59$ & 88 & 126 & 18 & 66 \\
\hline $60-69$ & 66 & 113 & 13 & 38 \\
\hline Median age in years & 58.0 & 57.0 & 57.0 & 56.0 \\
\hline $\begin{array}{l}\text { Occupation } \\
\text { professional/ } \\
\text { administrative }\end{array}$ & 24 & 29 & 1 & 5 \\
\hline clerical/sales & 63 & 138 & 22 & 71 \\
\hline blue-collar & 81 & 124 & 11 & 30 \\
\hline housewives & - & - & 2 & 13 \\
\hline Median years of education & 11.0 & 11.0 & 10.0 & 10.0 \\
\hline
\end{tabular}

All subjects were interviewed in person by trained interviewers. They were asked in detail about their drinking and smoking habits throughout their entire lives, past history of blood transfusion, etc. In this study, drinkers were defined as those who had drunk at least one or more units of alcoholic beverages per day, once a week or more, for at least one year. The unit ("drink" in the present study) corresponds to the amount of alcoholic beverage containing $23 \mathrm{ml}$ of ethanol, nearly the equivalent to $150 \mathrm{ml}$ of sake (rice wine), $90 \mathrm{ml}$ of shochu (a distilled alcoholic beverage made in Japan), $450 \mathrm{ml}$ of beer, and $60 \mathrm{ml}$ of hard liquor. Among the drinkers, those who had consumed $80 \mathrm{ml}$ or more ethanol per day for at least 10 years were regarded as having a history of "heavy drinking". For each study subject, the cumulative alcohol consumption in "drink-years" was calculated by multiplying the daily 
consumption of each type of alcoholic beverage expressed in "drinks" by the number of years of consumption and then by adding all types of beverages and the different periods of life. As for smoking habits, smokers were defined as those who had smoked almost every day, at least one pack (20 cigarettes) per week, for at least one year. Smokers who had given up smoking one or more years prior to interview were regarded as exsmokers. The cumulative amount of smoking in pack-years was then calculated for each subject.

Information on serum HBV markers was obtained from medical recorded for all cases. For the controls, sera were tested for hepatitis B surface antigen (HBsAg) by a reversed passive hemagglutination method. The sera from 91 cases and all the controls were stored at $-70^{\circ} \mathrm{C}$ and were available for anti-HCV testing. In addition, the sera from 75 patients with liver cirrhosis (LC), who met the above selection criteria b) and c) for the cases and who had no evidence of $\mathrm{HCC}$, were assayed for anti-HCV. Anti-HCV in the serum were detected by an enzyme-linked immunosorbent assay (ELISA, Ortho Diagnosis Systems, Raritan, NJ) using c100-3, and the sera positive in ELISA were retested by a recombinant immunoblot assay (RIBA, Chiron Corp., Emeryville, CA) using c100-3 and 5-1-1. ${ }^{(11)}$.

The relative risks (R.Rs) and their 95\% confidence intervals (CIs) were estimated by modeling the data through an unconditional logistic regression for controlling any possible confounding factors such as sex and age. ${ }^{(17)}$

\section{RESULTS AND DISCUSSION}

Table 3 demonstrates the prevalence of chronic HBV infection and past history of blood transfusion and the RRs for those factors. We confirmed the existence of a strong relationship between chronic HBV infection and HCC, as evidenced by the high RR of 14.6 among individuals positive for serum HBsAg after adjustment for sex and age. However, the prevalence of positive HBsAg was only $19 \%$ among HCC patients, which corresponded closely to the reported decline of HBV carriers shown in Table 1. For a history of blood transfusion, blood recipients with transfusions for causes related to liver diseases, e.g., due to bleeding from esophageal varices, were not included in the "positive" category. As displayed in Table 3, the past history of blood transfusion was significantly associated with around a three- to fourfold elevated HCC risk.

Table 3 Prevalence of chronic HBV infection and history of blood transfusion among study subjects and the relative risks of HCC for those factors

\begin{tabular}{|c|c|c|c|c|}
\hline \multirow{2}{*}{ Factor } & \multirow{2}{*}{ Sex } & \multicolumn{2}{|c|}{ Prevalence $(\%)$} & \multirow{2}{*}{$\mathrm{RR}^{\mathrm{a}}(95 \% \mathrm{CI})$} \\
\hline & & $\mathrm{HCC}$ & Controls & \\
\hline \multirow[t]{3}{*}{ HBsAg-positive } & Males & 16.7 & 2.4 & $9.3(3.9-22.5)$ \\
\hline & Females & 27.8 & 0.8 & $62.1(6.7-577.6)$ \\
\hline & Both & 18.6 & 2.0 & $14.6(6.4-33.3)$ \\
\hline \multirow{3}{*}{$\begin{array}{l}\text { Positive history of } \\
\text { blood transfusion }\end{array}$} & Males & 19.0 & 7.9 & $2.7(1.5-4.9)$ \\
\hline & Females & 30.6 & 10.1 & $4.2(1.6-10.8)$ \\
\hline & Both & 21.1 & 8.5 & $3.0(1.8-4.9)$ \\
\hline
\end{tabular}

$a_{R R}$ with those negative for the corresponding factor as the referent and adjusted for age (and sex in both sexes). $b_{B l o o d}$ recipients due to liver diseases were regarded as non-reciplents (see text). 
The RRs of HCC for drinking habits and their $95 \%$ CIs are represented in Figure 1. In males, $131 \mathrm{HCCs}(78 \%)$ and 218 controls $(75 \%)$ had some history of drinking, and 52 HCCs $(31 \%)$ and 59 controls $(20 \%)$ had a positive history of heavy drinking; in females, 9 HCCs (25\%) and 17 controls (14\%) had some history of drinking, and 2 HCCs $(6 \%)$ and 4 controls $(3 \%)$ had a positive history of heavy drinking. The risk increase among those who had some history of drinking, as compared with nondrinkers, was slight and statistically nonsignificant. However, the positive history of heavy drinking was associated with a significantly increased RR of 1.8 .

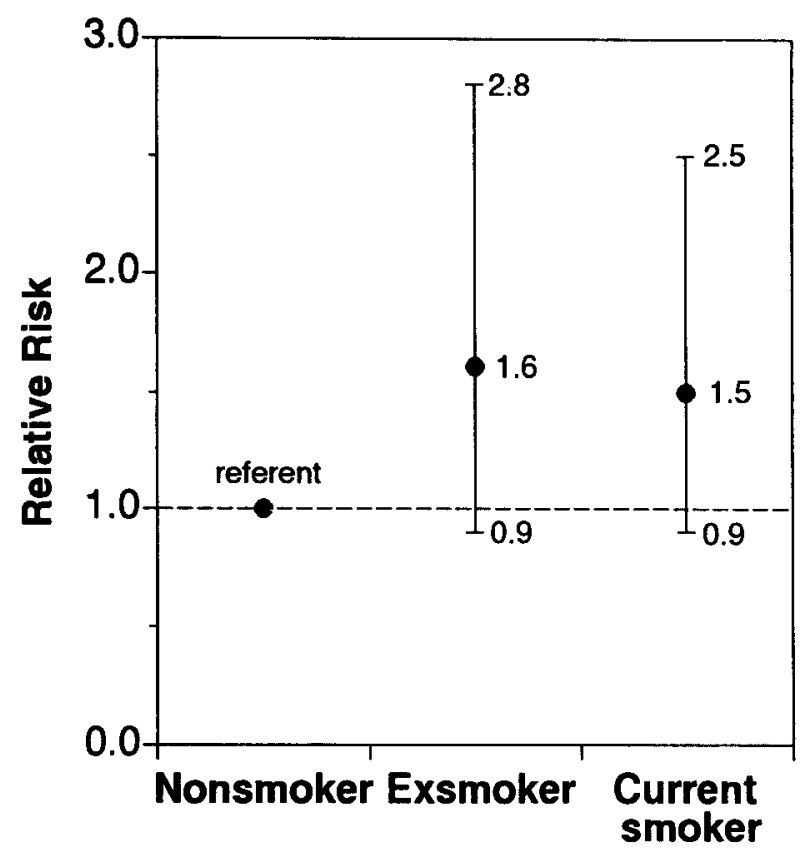

Figure 1 Relative risks of HCC according to drinking habits, adjusted for sex and age. Error bars show $95 \%$ confidence intervals. Regarding the definition of those who had some history of drinking and positive history of heavy drinking, see text.

As for smoking habits, the proportions of nonsmokers, exsmokers and current smokers in males were $10 \%, 29 \%$ and $61 \%$, respectively, for $\mathrm{HCCs}$, and $17 \%, 26 \%$ and $56 \%$, respectively, for the controls; the corresponding figures in females were $81 \%, 8 \%$ and $11 \%$ for HCCs, and $82 \%, 5 \%$ and $13 \%$ for controls. The sex and age adjusted RRs were estimated to be 1.6 for exsmokers and 1.5 for current smokers, as compared to nonsmokers (Figure 2). Both estimate exceeded unity, but were not significantly elevated.

The association of drinking and smoking habits with HCC was also examined in terms of the cumulative consumption of alcohol and cigarettes. The subjects were classified into nine exposure categories according to tertiles of the cumulative alcohol consumption and tertiles of the cumulative amount of smoking among male controls. The RR for each category, as compared with the lowest consumption category, is demonstrated in Figure 3. Categories with the highest alcohol consumption showed RRs of around 2 regardless of cigarette consumption. 
Meanwhile, the effect of cigarette consumption was not evident within each level of alcohol consumption. A test for any interaction between these factors was not significant $(p=0.87)$.

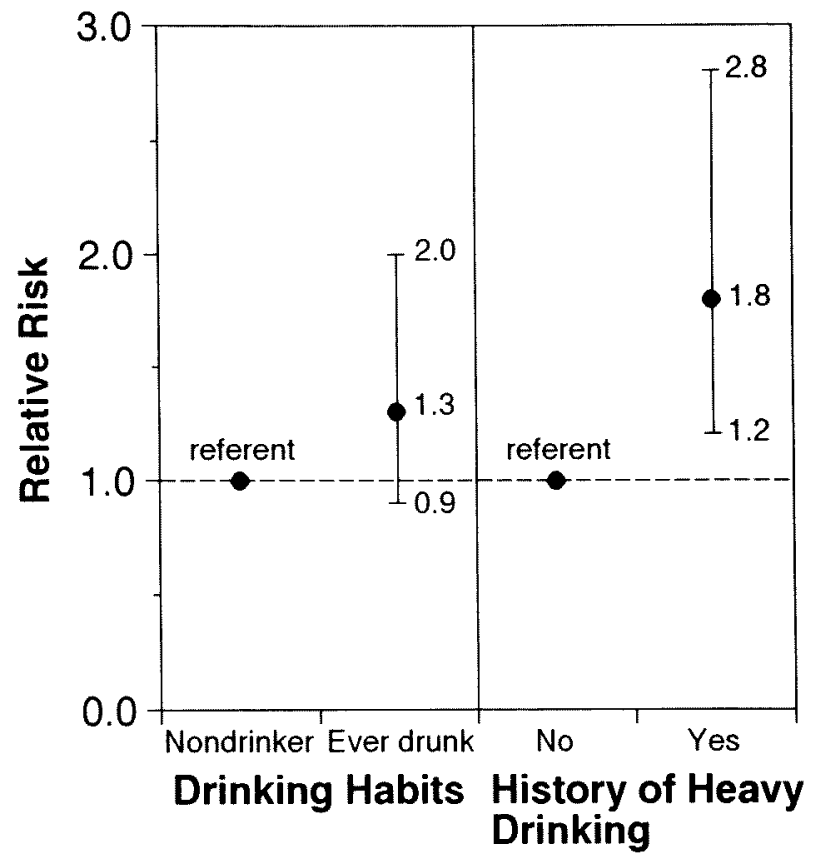

Figure 2 Relative risks of HCC according to smoking habits, adjusted for sex and age. Error bars show $95 \%$ confidence intervals. Regarding the definition of ex- and current smokers, see text.

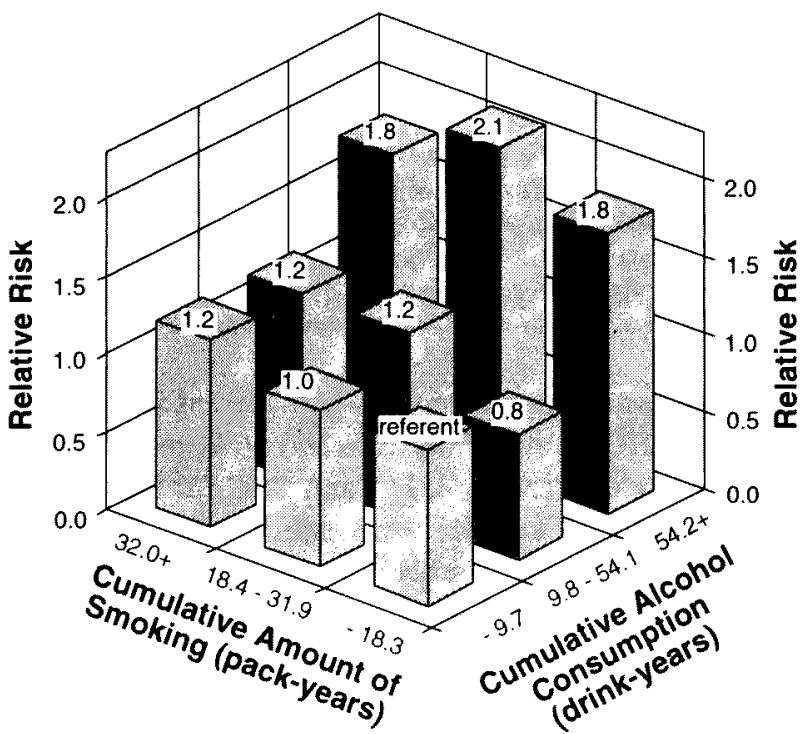

Figure 3 Relative risks of $\mathrm{HCC}$ according to cumulative alcohol consumption and cumulative amount smoking, adjusted for sex, age, HBsAg status, history of blood transfusion and family history of liver diseases. The cumulative measures were categorized by tertiles among male controls. Regarding the derivation of cumulative alcohol consumption in "drink-years", see text. 
Figure 4 shows the prevalence of anti-HCV among 91 patients with $\mathrm{HCC}, 75$ patients with LC and 410 controls, from whom sera were available for testing anti-HCV. Anti-HCV were detected in $51 \%$ of HCC, $51 \%$ of LC and $3 \%$ of the controls by both ELISA and RIBA. There were also very few subjects that were ELISA positive and RIBA non-reactive. RIBA is known to be more specific than ELISA. ${ }^{(18,19)}$ Although some false positives by ELISA are suspected, especially when using stored sera ${ }^{(20)}$, our results suggest that, in ELISA, the higher prevalence of anti-HCV among HCCs and LCs than among controls is not due to false positives. Only ELISA-positive and RIBA-reactive results are regarded as positive for anti-HCV in the subsequent analyses.

Figure 5 displays the prevalence of anti-HCV by selected risk factors. Since the number of female subjects was limited, only the results of male subjects are represented. Among the HBsAg-positive subjects, the prevalence of anti-HCV ranged from 0 to $20 \%$. These values were much lower than those shown in Figure 4. Among the blood recipients, anti-HCV positives made up about $70 \%$ of the patients with HCC or LC and only $13 \%$ among the controls. One notable finding was the high prevalence of anti-HCV positives among heavy drinkers or persons without any factors in the patients with HCC or LC. Forty-six to $75 \%$ of those patients were positive for anti-HCV. Although HCV was thought to be a blood transfusion-related agent at first, our data suggest the presence of other routes of transmission. In addition, HCV may play a role in patients with so-called "alcoholic" cirrhosis.

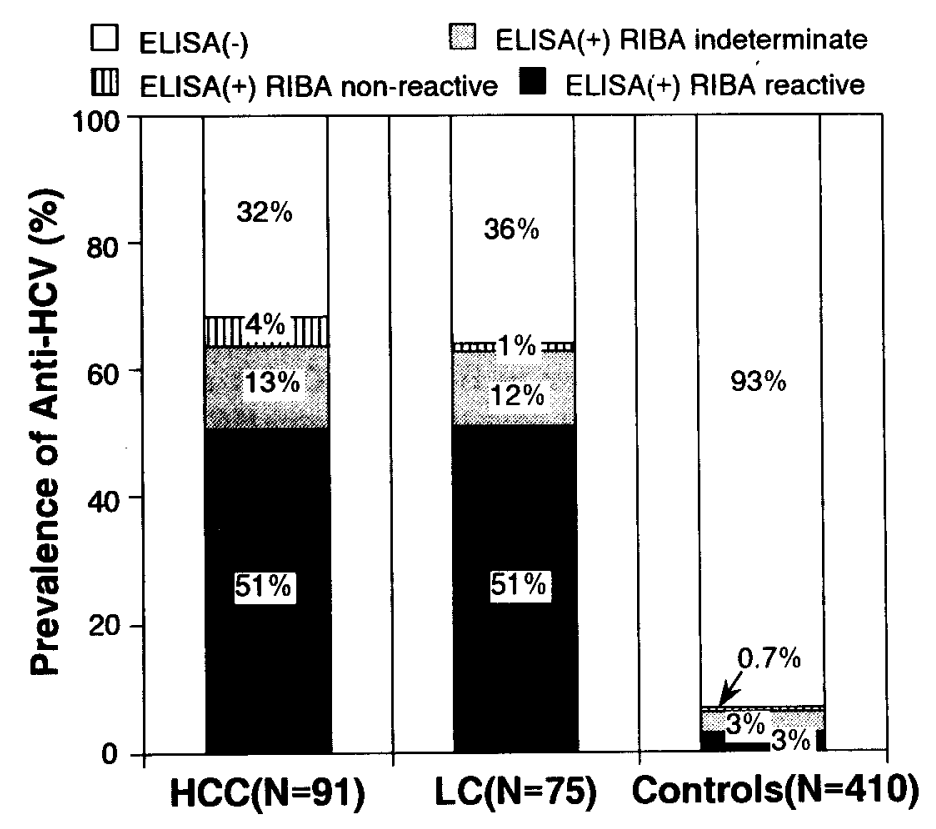

Figure 4 Prevalence of anti-HCV among study subjects according to results from ELISA and RIBA 


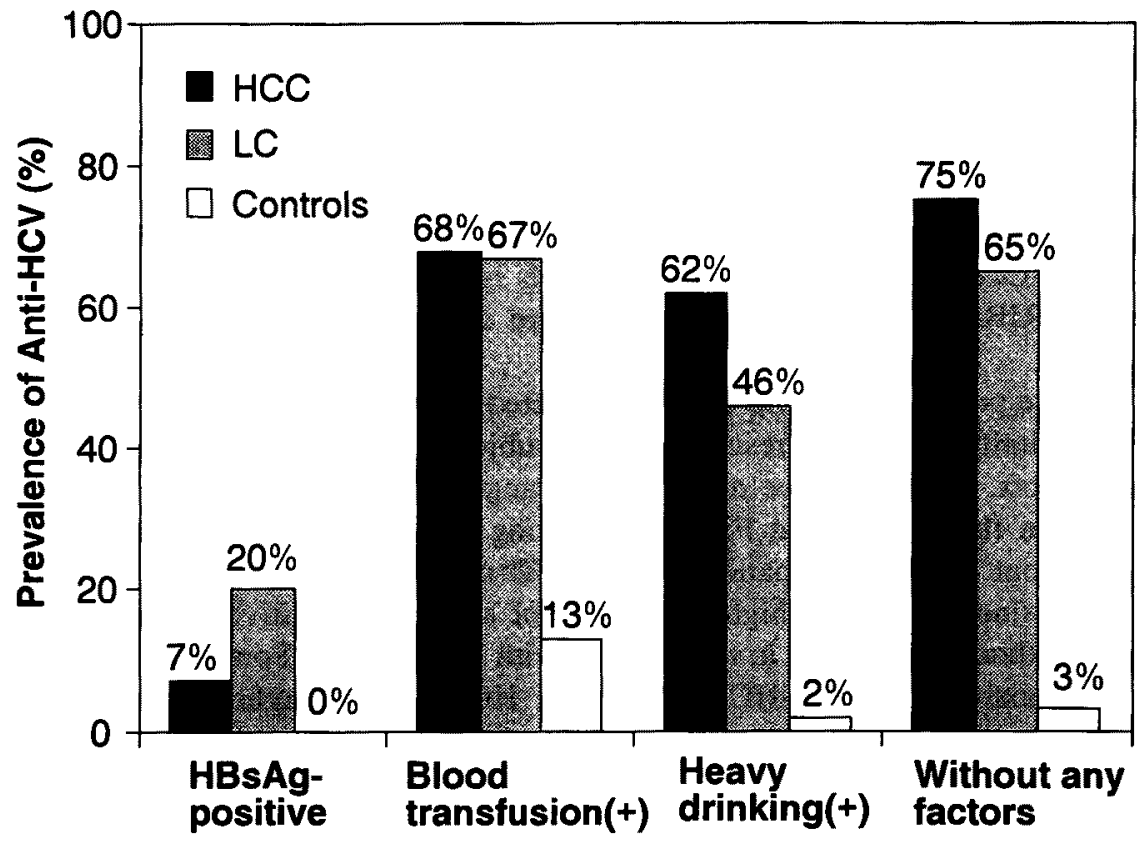

Figure 5 Prevalence of RIBA anti-HCV among male subjects according to selected risk factors. Blood recipients due to causes related to liver diseases were not included in the positive category of blood transfusion. Regarding the definition of heavy drinking, see text.

The RRs for selected factors were estimated with and without adjustment for anti-HCV (Table 4). Since these RR estimates are based only on the subjects with a proven anti-HCV status, they slightly differ from the RRs derived from the previous analyses. Our study revealed an extremely strong association of anti-HCV with both $\mathrm{HCC}$ and LC; the RRs among anti-HCV positive subjects were 52.3 for HCC and 64.4 for LC. These values far exceeded the RRs of 15.3 or 6.1 for positive HBsAg. Among blood recipients, the RRs were 3.8 for $\mathrm{HCC}$ and 4.7 for LC. After adjustment for anti-HCV, these figures decreased to 2.0 and 2.7 , respectively. There was about a two-fold increase in HCC or LC risk among heavy drinkers; this association did not change materially after adjustment for anti-HCV.

Table 4 Relative risks (with 95\% confidence intervals) of HCC and LC for selected risk factors with and without adjustment for RIBA anti-HCV

\begin{tabular}{|c|c|c|c|c|c|c|c|c|}
\hline \multirow{3}{*}{\multicolumn{2}{|c|}{ Factor }} & \multicolumn{4}{|c|}{$\mathrm{RR}^{\mathrm{a}}(95 \times \mathrm{Cl})$ of $\mathrm{HCC}$} & \multicolumn{3}{|c|}{$R^{a}(95 \times C I)$ of $L C$} \\
\hline & & \multicolumn{2}{|c|}{ ant I-HCr } & \multirow{2}{*}{\multicolumn{2}{|c|}{$\begin{array}{l}\text { antl-HCV } \\
\text { adjusted }\end{array}$}} & \multicolumn{2}{|c|}{ anti-HCV } & \multirow{2}{*}{$\begin{array}{l}\text { antl-HCV } \\
\text { adjusted }\end{array}$} \\
\hline & & not & adjusted & & & not & adjusted & \\
\hline \multirow[t]{2}{*}{ ant $1-\mathrm{HCV}$} & Indeterminate & 11.8 & $(4.8-28.8)$ & & - & 10.4 & $(3.9-28.1)$ & - \\
\hline & reactive & 52.3 & $(23.9-114.3)$ & & - & 64.4 & $(27.4-151 \cdot 4)$ & - \\
\hline \multicolumn{2}{|c|}{ HBSAE positive } & 15.3 & $(6.1-38.1)$ & & $b$ & 6.1 & $(2.2-17.2)$ & $\mathbf{b}$ \\
\hline \multicolumn{2}{|c|}{$\begin{array}{l}\text { Positive history of } \\
\text { blood transfusion }\end{array}$} & 3.8 & $(2.0-7.0)$ & 2.0 & $(0.9-4.7)$ & 4.7 & $(2.5-8.8)$ & $2.7(1.2-6.2\}$ \\
\hline \multicolumn{2}{|c|}{$\begin{array}{l}\text { Positive history of } \\
\text { heavy drinking }\end{array}$} & 2.2 & $(1.3-3.8)$ & 2.1 & $(1.0-4.4)$ & 1.4 & $(0.7-2.8)$ & $1.8(0.7-4.3)$ \\
\hline
\end{tabular}

${ }^{a_{R R}}$ adjusted for sex. age, occupation and years of education. For antl-HCV, Indivlduals with ELISA negative or RIBA non-reactive results as the referent. For the other factors, those negative for the corresponding factor as the referent. $b_{R R}$ estimate was extremely biased due to a highy negative correlation between HBsAg status and anti-HCV status. 
In conclusion, we detected a strong relationship between chronic HBV infection and HCC. A past history of blood transfusion was also a significant risk factor for HCC. Heavy alcohol consumption was moderately associated with an increased risk of $\mathrm{HCC}$, but the association with cigarette smoking was uncertain and, if any at all, relatively weak. No interaction between drinking and smoking habits on $\mathrm{HCC}$ risk was observed in this study. The association of $\mathrm{HCV}$ infection with $\mathrm{HCC}$ was found to be extremely strong, and the etiological role of $\mathrm{HCV}$ is probably more important than chronic HBV infection. Attributable risk calculations suggest that chronic $\mathrm{HBV}$ infection, heavy drinking and $\mathrm{HCV}$ infection may account for $17 \%$ (95\% CI 12-23), 13\% (95\% CI 4-22), and 49\% (95\% CI 39-60) of HCC occurrences, respectively, in Fukuoka prefecture. We conclude that the identification of infectious routes of HCV other than blood transfusion will be important in the prevention of HCC and, therefore, further investigations appear to be warranted.

\section{ACKNOWLEDGEMENTS}

We are grateful to T. Hayashi, N. Hashimoto and M. Matsushige for interviewing subjects; to I. Hirohata of the Department of Public Health, Kurume University School of Medicine, for preparing questionnaires and advice on our interviews; and to B.T. Quinn from Kyushu University for proofreading the manuscript. We also express our deep appreciation to $S$. Takeshita of the Department of Information and Management Science, Tokai University Fukuoka Junior College; to Dr. S. Koga of the lizuka Hospital; to Drs. K. Sugimachi and S. Kitano of the Second Department of Surgery, Drs. H. Nawata, S. Sakamoto, B. Ando and H. Miyata of the Third Department of Internal Medicine, and Dr. H. Ishibashi of the First Department of Internal Medicine, Kyushu University School of Medicine; to Dr. T. Kanematsu of the Second Department of Surgery, Nagasaki University School of Medicine; to Dr. F. Ohryohji of the Health Promotion Section, Bureau of Public Health of Fukuoka City; to Drs. Y. Maeda and H. Kiyokawa, K. Tokunaga and Y. Irita of the Fukuoka Red Cross Blood Center; to Drs. Y. Arase and N. Nishino of the Hakata Public Health Center; and to the staff of all the relevant departments for their kind cooperation.

\section{REFERENCES}

1. Statistics and Information Department, Minister's Secretariat, Ministry of Health and Welfare: Mortality Statistics from Malignant Neoplasms 1972-1984, Special Report of Vital Statistics in Japan. Ministry of Health and Welfare, Tokyo, 1986 (in Japanese).

2. Liver Cancer Study Group of Japan: Survey and follow-up study of primary liver cancer in Japan - Report 8 -. Acta Hepatol. Jpn., 29: 1619, 1988 (in Japanese).

3. Liver Cancer Study Group of Japan: Survey and follow-up study of primary liver cancer in Japan - Report 4 -. Acta Hepatol. Jpn., 20: 433, 1979 (in Japanese).

4. IARC Monographs on the evaluation of carcinogenic risks to humans, Vol. 44: alcohol drinking. IARC, Lyon, 1988.

5. Trichopoulos, D., Macmahon, B., Sparros, L. et al.: Smoking and hepatitis B-negative primary hepatocellular carcinoma. J. Natl. Cancer Inst., 65: 111, 1980.

6. Trichopoulos, D., Day, N.E., Kaklamani, E. et al.: Hapatitis B virus, tobacco smoking and ethanol consumption in the etiology of hepatocellular carcinoma. Int. J. Cancer, 39: 45, 1987. 
7. Yu, H., Harris, R.E., Kabat, G.C., et al.: Cigarette smoking, alcohol consumption and primary liver cancer: a case-control study in the USA. Int. J. Cancer, 42: 325, 1988.

8. Hirayama, T.: A large-scale cohort study on risk factors for primary liver cancer, with special reference to the role of cigarette smoking. Cancer Chemother. Pharmacol., 23: $114 \mathrm{~S}, 1989$.

9. Kiyosawa, K., Akahane, Y., Nagata, A. et al.: Hepatocellular carcinoma after non-A, non-B posttransfusion hepatitis. Am. J. Gastroenterol., 79: 777, 1984.

10. Choo, Q.-L., Kuo, G., Weiner, A.J. et al.: Isolation of a cDNA clone derived from a bloodborne non-A, non-B viral hepatitis genome. Science, 244: 359, 1989.

11. Kuo, G., Choo, Q.-L., Alter, H.J. et al.: An assay for circulating antibodies to a major etiologic virus of human non-A, non-B hepatitis. Science, 244: 362, 1989.

12. Saito, I., Miyamura, T., Ohbayashi, A. et al.: Hepatitis $C$ virus infection is associated with the development of hepatocellular carcinoma. Proc. Natl. Acad. Sci., USA, 87: 6547, 1990.

13. Nishioka, K., Watanabe, J., Furuta, S. et al.: A high prevalence of antibody to the hepatitis $\mathrm{C}$ virus in patients with hepatocellular carcinoma in Japan. Cancer, 67: 429, 1991.

14. Tanaka, K., Hirohata, T., Takashita, S.: Blood transfusion, alcohol consumption, and cigarette smoking in causation of hepatocellular carcinoma: a case-control study in Fukuoka, Japan. Jpn. J. Cancer Res. (Gann), 79: 1075, 1988.

15. Tanaka, K., Hirohata, T., Koga, S. et al.: Hepatitis C and hepatitis B in the etiology of hepatocellular carcinoma in the Japanese population. Cancer Res., 51: 2842, 1991.

16. Tanaka, K., Hirohata, T., Takashita, S. et al.: Hepatitis B virus, cigarette smoking and alcohol consumption in the development of hepatocellular carcinoma: a case-control study in Fukuoka, Japan. Int. J. Cancer, in press.

17. Breslow, N.E., Day, N.E.: Statistical Methods in Cancer Research, Vol. 1: The Analysis of Case-Control Studies. IARC, Lyon, 1980.

18. Ebeling, F., Naukkarinen, R., Leikola, J.: Recombinant immunoblot assay for hepatitis C virus antibody as predictor of infectivity. Lancet, i: 982, 1990.

19. Weiner, A.J., Truett, M.A., Rosenblatt, J. et al.: HCV testing in low-risk population. Lancet, ii: 695, 1990.

20. Johnson, P.J., Williams, R.: Hapatitis C antibodies and hepatocellular carcinoma: new clues or a false trail? J. Natl. Cancer Inst., 82: 986, 1990. 